\title{
Desempenho Larval do Camarão-d'Água-Doce (Macrobrachium rosenbergii De Man, 1879) Submetido a Diferentes Regimes Alimentares
}

\author{
Luciana Almada Thomaz ${ }^{2}$, Lídia Miyako Yoshii Oshiro ${ }^{3}$, Andrea Cecchetto Bambozzi ${ }^{4}$, \\ José Teixeira de Seixas Filho 5
}

RESUMO - O desempenho das larvas de Macrobrachium rosenbergii submetidas a quatro diferentes regimes alimentares foi verificado utilizando a observação diária dos subestádios larvais e as primeiras metamorfoses. As larvas foram estocadas em densidade

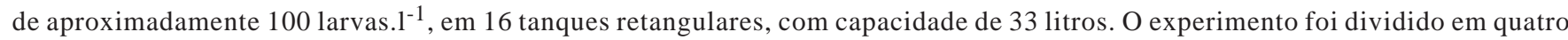
unidades experimentais (blocos), nos quais foram testados quatro regimes alimentares na larvicultura em circuito aberto, com quatro repetições por tratamento. Os regimes alimentares substituindo progressivamente náuplio de Artemia sp. (nas) pelo rotífero Brachionus plicatilis (rots) foram os seguintes tratamentos: 100\% Brachionus plicatilis (30 rots/mL) (T1); 100\% Artemia (5 nas/ mL) (T2), 60\% Artemia (3 nas $/ \mathrm{mL})+40 \%$ Brachionus plicatilis (12 rots/ mL) (T3) e 40\% Artemia (2 nas $/ \mathrm{mL})+60 \%$ Brachionus plicatilis (18 rots/ mL) (T4), sendo adicionada a estes tratamentos ração úmida. Os resultados da mudança dos subestádios larvais demonstraram que não houve diferença significativa entre os tratamentos T2, T3 e T4. No tratamento T1 (100\% Brachionus plicatilis) houve mortalidade total no $14^{\circ}$ dia do experimento. O efeito da ocorrência das primeiras metamorfoses para pós-larvas, ocorreu ao 270 o dia nos tratamentos T2, T3 e T4. Conseqüentemente, o ciclo de larvicultura até a metamorfose de 90\% para pós-larva (PL1) foi o mesmo nos tratamentos (35 dias). Portanto, a utilização do rotífero enriquecido e congelado no regime alimentar das larvas permitiu desempenho satisfatório em termos de desenvolvimento larval de M. rosenbergii até a passagem para o estádio de pós-larva.

Palavras-chave: aqüicultura, alimentação, Brachionus plicatilis, camarão-d'água-doce, larvicultura, Macrobrachium rosenbergii

\section{Larval Performance of the Freshwater Prawn (Macrobrachium rosenbergii De Man, 1879) Submitted to Different Feeding Systems}

\begin{abstract}
The feeding is one of the most important factors to larval development and the sucess of the commercial cultivation of decapod crustacean larvae depends on the efficient and economic utilization of the available food. The performance of Macrobrachium rosenbergii larva submitted to four different feeding systems was tested by using the daily observation of the larval substages and the first metamorphoses. The larvae were stocked in a density of approximately 100 larvas..$^{-1}$, in 16 tanks, with 33 liters capacity. The experiment was divided in four experimental units (blocks). Four feeding systems were tested in the hatchering in an open circuit, with four replicates for treatment. The feeding systems in which progressive substitutions of the nauplii of Artemia sp. (nas) were accomplished, for the rotifer Brachionus plicatilis (rots), the following treatments were tested: $100 \%$ Brachionus plicatilis (30 rots/mL) (T1); 100\% Artemia (5 nas/mL) (T2), 60\% Artemia (3 nas / mL) + 40\% Brachionus plicatilis (12 rots/mL) (T3) and $40 \%$ Artemia $(2 \mathrm{nas} / \mathrm{mL})+60 \%$ Brachionus plicatilis (18 rots $/ \mathrm{mL}$ ) (T4), Humid ration was added to all treatments. The results of the change of the larval stage demonstrated that there was not significant difference among the treatments T2, T3 and T4. The treatment T1 (100\% Brachionus plicatilis) had total mortality in the $14^{\text {th }}$ day of the experiment. The effect of the occurrence of the first metamorphoses for post-larvae, ocurred at 27th day in the treatments T2, T3 and T4. Consequently, the hatchering cycle to the metamorphosis of $90 \%$ for post-larvae (PL1), was the same in the respective treatments (35 days). Therefore, the use of the enriched rotifer and frozen in the feeding systems of the larvae allowed a satisfactory performance in terms of larval development of $M$. rosenbergii until the passage for the post-larvae stage.
\end{abstract}

Key Words: aquaculture, food, Brachionus plicatilis, freshwater prawn, hatchery, Macrobrachium rosenbergii

\footnotetext{
${ }^{1}$ Parte da Dissertação de Mestrado em Zootecnia apresentada pelo primeiro autor à UFRRJ.

${ }^{2}$ Eng. Agrônoma, aluna do Programa de Pós-graduação em Zootecnia do Instituto de Zootecnia UFRRJ (almadalt@yahoo.com.br).

3 Professora Adjunto 4 - Departamento de Produção Animal. Instituto de Zootecnia da UFRRJ (oshiro@ufrrj.br).

${ }^{4}$ Zootecnista - aluna do Programa de Pós-graduação em Zootecnia do Instituto de Zootecnia UFRRJ (abambozzi@yahoo.com.br).

${ }^{5}$ Biólogo, D.Sc. em Zootecnia. Pesquisador FIPERJ (joseseixas@fiperj.rj.gov.br).
} 


\section{Introdução}

A criação de camarão-d'água-doce é um dos setores da aqüicultura que mais cresce no mundo. Segundo os dados da FAO (2002), de 1990 a 2000, o volume de $M$. rosenbergii produzido passou de 21.000 para 118.500 toneladas e estima-se que, na virada do milênio, a produção ultrapassou 200.000 t, movimentando mais de US\$ 1 bilhão (New, 2000). Estes dados indicam que o setor cresceu mais de 1000\% na última década (Valenti, 2001).

A obtenção de pós-larvas é um dos principais obstáculos ao desenvolvimento de cultivos comerciais de camarão-d'água-doce, por ser uma atividade dependente de alimentos vivos que requerem cuidados e monitoramento freqüente, sendo a alimentação um dos fatores mais importantes no desenvolvimento larval (Yufera et al., 1985), pois o estabelecimento de adequado sistema de alimentação durante a fase larval do camarão-d'água-doce, com relação à ingestão, percepção, captura, apreensão e ingestão dos alimentos inertes e vivos, proporciona bom desenvolvimento em cada estádio larval (Barros \& Valenti, 1997, 2003).

Na larvicultura, a administração de uma alimentação adequada é fundamental, pois afeta diretamente a sobrevivência e o tempo de desenvolvimento larval. Portanto, é importante que mais de um alimento de mesma natureza seja utilizado para que o número de aminoácidos, vitaminas e demais nutrientes seja o mais completo possível (Valenti, 1991, 1998).

Várias fontes de alimento (vivo ou congelado) têm sido experimentadas em larviculturas de peixes e crustáceos, visando estabelecer uma dieta adequada. Entretanto, o mais utilizado na larvicultura de camarão-d'água-doce é o náuplio do microcrustáceo Artemia sp., graças à sua praticidade e ao seu valor nutritivo (Sorgeloos et al., 1983).

Silva \& Rodrigues (1997) relataram que o uso de náuplios do microcrustáceo Artemia sp. como alimento vivo não tem sido inteiramente livre de transtornos. Atualmente, a mudança do clima em todo o mundo agrava esse problema, reduzindo o número de regiões produtoras de cistos e acarretando sérios problemas aos aqüicultores, com a redução da oferta e conseqüente aumento no preço de mercado (Lavens et al., 2000).

A produção de cistos de Artemia sp. tem apresentado incremento de $15 \%$ ao ano, totalizando uma participação de US\$ 1,5 a 2,5 no custo de produção por milheiro de pós-larvas, embora a produção atual não tenha atendido a demanda mundial de Artemia sp. (Alam et al., 1991, 1993a, 1993b), contribuindo para o alto custo dos cistos deste microcrustáceo, tornando-se necessário pesquisar fontes alternativas de alimentos vivos com valores nutritivos semelhantes, porém com preços mais acessíveis (Lai, 1985).

Buscando o sucesso no cultivo comercial de larvas de $M$. rosenbergii e maior autonomia no cultivo de alimentos vivos, além do barateamento na produção larval do camarão com a substituição da Artemia sp., vários organismos aquáticos vêm sendo estudados visando obter novas fontes protéicas (Seixas Filho et al., 1984, 2000; Lovett \& Felder, 1988; Watanabe et al., 1989; Sorgeloos \& Léger, 1992; Stottrup \& Attramadal, 1992; Silva \& Rodrigues, 1997). Contudo, não forneceram resultados satisfatórios quando comparados com a Artemia sp. (New et al., 1990, 1995, 2000a; Correia et al., 2000).

Neste contexto, conduziu-se o presente trabalho com o objetivo de avaliar o desempenho das larvas do camarão-d'água-doce ( $M$. rosenbergii), por meio da observação diária dos subestádios larvais e das primeiras metamorfoses, quando submetidos à substituição total e/ou parcial dos náuplios de Artemia sp., pelo rotífero Brachionus plicatilis enriquecido e congelado.

\section{Material e Métodos}

Este trabalho foi executado no Laboratório da Estação de Aqüicultura Almirante Paulo Moreira (E.A.A.P.M), da Fundação do Instituto de Pesca do Estado do Rio de Janeiro (FIPERJ). O experimento foi realizado no período de 11 de março de 2001 a abril de 2001, com larvas de camarão-d'água-doce (M. rosenbergii) eclodidas de várias fêmeas ovígeras, oriundas da Fazenda Santa Helena, Silva Jardim (RJ).

As larvas do camarão-d'água-doce, foram esto-

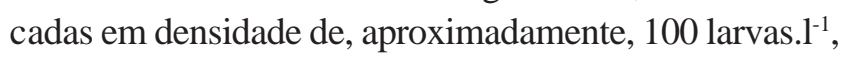
em 16 tanques retangulares, com capacidade individual de 33 litros. Os tanques foram revestidos em seu interior com tinta epóxi preta, para impedir a fixação de agentes contaminantes e facilitar a visualização e apreensão do alimento (náuplios de Artemia sp., rotífero enriquecido e partículas de ração) pelas larvas de camarão. Os fatores físico-químicos da água dos tanques de larvicultura foram monitorados durante todo o período experimental. Para o valor do

R. Bras. Zootec., v.33, n.6, p.1934-1941, 2004 (Supl. 2) 
pH foi observada variação média de 8,0 a 8,3 ppm. Os níveis de amônia mantiveram-se inferiores a 0,1ppm e o oxigênio manteve-se sempre próximo à saturação (7,5-8,0 ppm). A temperatura foi mantida em $28^{\circ} \mathrm{C}\left( \pm 1 \mathrm{C}^{\circ}\right)$, com auxílio de aquecedores acoplados a termostato, e a salinidade foi mantida a $16 \mathrm{ppm}$.

Desde o primeiro dia de criação, foi fornecido às larvas alimento inerte (ração úmida), administrado ad libitum de forma quantitativa, equivalente para todos os tratamentos. A ração foi fornecida três vezes ao dia (às 8, 10 e $12 \mathrm{~h}$ ), enquanto o alimento vivo foi fornecido às $17 \mathrm{~h}$, após a limpeza dos tanques.

Cada unidade experimental recebeu aeração constante, por meio de soprador elétrico e mangueiras plásticas 3/16" providas de pedras porosas em suas extremidades, reguladas por registro de mesmo calibre, mantendo-se o nível de oxigênio próximo à saturação. Esta aeração serviu também para manter as partículas alimentares em suspensão, proporcionando distribuição homogênea das larvas por toda coluna d'água e evitando, assim, aumento da taxa de canibalismo.

A limpeza dos tanques foi feita diariamente às $13 \mathrm{~h}$, por sifonamento, retirando-se a aeração, aguardando decantação mais concentrada das partículas. Em seguida, com o uso de uma mangueira plástica transparente de $3 / 4$ " sifonavam-se as sobras de alimento, de matéria orgânica e de fezes das larvas concentradas no fundo do tanque para baldes de fibra de vidro providos de tela de $150 \mu \mathrm{m}$ para retenção das larvas que se encontravam próximas ao fundo. Efetuada esta operação, recolocava-se a aeração, sendo $90 \%$ do volume da água dos tanques renovados e as larvas eventualmente sifonadas devolvidas ao sistema.

Alternadamente, dez larvas de cada unidade experimental (tanque) (total de 160 larvas) foram coletadas ao acaso para o acompanhamento do desenvolvimento larval, a partir das variáveis índice de estádio larval e estádio de repleção.

a. Índice de estádio larval - os estádios larvais foram observados de acordo com o critério desenvolvido por Uno \& Soo (1969). Como forma de padronização internacional neste trabalho, será referenciado com a sigla LSI (Larval Stage Index), que foi calculado pela média ponderada dos estádios larvais e obtido por meio das observações dos estádios larvais diários (Daniels et al., 1992). b. Estádio de repleção - foi verificada a quantidade de alimento ingerido pelas larvas amostradas por meio de observações visuais da quantidade de alimento no trato digestivo, utilizando-se a seguinte escala arbitrária: vazio (V); parcialmente cheio (M) e cheio (C); e calculada a média ponderada das observações diárias (Rodrigues et al., 1998).

Ao final do experimento, quando 90\% das larvas completaram a metamorfose e passaram à fase de pós-larva, a salinidade da água foi gradativamente reduzida a zero e o experimento foi encerrado, obtendo-se os dados de tempo de larvicultura e a taxa de metamorfose.

O experimento foi conduzido em blocos ao acaso, nos quais foram testados quatro regimes alimentares na larvicultura (em circuito aberto do camarão $M$. rosenbergii), com quatro repetições por tratamento. Nos regimes alimentares em que foram feitas substituições progressivas do náuplios (nas) de Artemia sp. pelo rotífero (rots) B. plicatilis, foram testados os seguintes tratamentos: $100 \%$ B. plicatilis (30 rots $/ \mathrm{mL}$ ) (T1); 100\% Artemia sp. (5 nas/mL) (T2), 60\% Artemia sp. (3 nas $/ \mathrm{mL})+40 \%$ B. plicatilis (12 rots $/ \mathrm{mL}$ ) (T3) e $40 \%$ Artemia sp., (2 nas $/ \mathrm{mL})+60 \%$ B. plicatilis (18 rots/mL) (T4), sendo adicionada a estes tratamentos ração úmida ad libitum de forma quantitativa, equivalente para todos os tratamentos. Os valores de substituição utilizados foram baseados nos estudos de Seixas Filho et al. (1984), nos quais foram utilizados 30 rotíferos para cada cinco náuplios de Artemia sp., para efeito de equivalência.

O método não-paramétrico do qui-quadrado foi utilizado para a análise estatística dos resultados do índice de estádio larval e do estádio de repleção.

\section{Resultados e Discussão}

Os estádios do desenvolvimento larval verificado em dias alternados demonstraram a ocorrência da metamorfose (transformação das larvas em póslarvas) aos 35 dias nos tratamentos T2, T3 e T4 (Figura 1).

O índice de estádios larvais do tratamento T1, no 6o dia de experimento, apresentou a maioria das larvas no estádio III, enquanto, em T2 T3 e T4, estavam no estádio V (Figura 1; Tabela 1), e pode ser indicativo de que os rotíferos enriquecidos e congelados não foram suficientes em termos nutricionais na 
dieta dessas larvas. Durante este período, as larvas submetidas a este tratamento apresentaram o estômago parcialmente ou completamente vazio, o que pode estar relacionado a dificuldades na captura dos rotíferos congelados (Tabela 1).

Após o VI estádio larval, do 14ํㅜ dia de criação em diante, os resultados indicaram que os índices de estádio larval e índices de estádio de repleção não variaram entre os tratamentos T3 e T4, quando comparados ao T2 (Tabela 1). Esta proximidade de resultados indica tendência de maior aceitação do rotífero enriquecido e congelado e/ou maior facilidade de apreensão e, por conseguinte, a importância desse alimento na nutrição das larvas nos estádios intermediários e finais da larvicultura. Outros experimentos talvez possam confirmar esta tendência e sugerir que o rotífero enriquecido e congelado Brachionus plicatilis deva ser utilizado a partir do VI estádio larval.

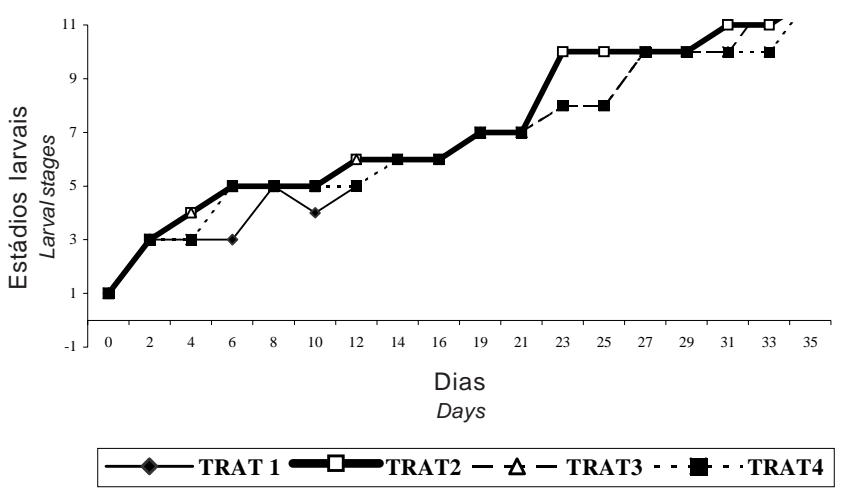

Figura 1 - Desenvolvimento larval do camarão-d'águadoce ( $M$. rosenbergii) submetido aos diferentes tratamentos alimentares, ao final do período experimental (35 dias).

Figure 1 - Larval development of the fresh water prawn M. rosenbergii submitted to different feeding systems, at the end of the experimental period (35 days).

Tabela 1 - Dados referentes ao desenvolvimento larval e ao estádio de repleção das larvas em cada tratamento

Table 1 - Data of the larval development and the larvae repletion stadium in each treatment

\begin{tabular}{|c|c|c|c|c|c|c|c|c|}
\hline \multirow[b]{3}{*}{$\begin{array}{l}\text { Período } \\
\text { Period }\end{array}$} & \multicolumn{8}{|c|}{$\begin{array}{l}\text { Tratamentos } \\
\text { Treatments } \\
\end{array}$} \\
\hline & \multicolumn{2}{|c|}{$\begin{array}{c}\mathrm{T} 1 \\
(100 \% \text { Brachionus } \\
\text { plicatilis })\end{array}$} & \multicolumn{2}{|c|}{$\begin{array}{c}\mathrm{T} 2 \\
(100 \% \text { Artemia sp.) }\end{array}$} & \multicolumn{2}{|c|}{$\begin{array}{c}\text { T3 } \\
(60 \% \text { Artemia sp. }+40 \% \\
\text { Brachionus plicatilis) } \\
\end{array}$} & \multicolumn{2}{|c|}{$\begin{array}{c}\text { T4 } \\
(40 \% \text { Artemia sp. }+60 \% \\
\text { Brachionus plicatilis })\end{array}$} \\
\hline & $\begin{array}{l}\text { Índice } \\
\text { estádios } \\
\text { larvais } \\
\end{array}$ & $\begin{array}{l}{ }^{*} \text { Estádio de } \\
\text { repleção }\end{array}$ & $\begin{array}{c}\text { Índice } \\
\text { estádios } \\
\text { larvais } \\
\end{array}$ & $\begin{array}{l}\text { Estádio de } \\
\text { repleção }\end{array}$ & $\begin{array}{l}\text { Índice } \\
\text { estádios } \\
\text { larvais } \\
\end{array}$ & $\begin{array}{l}\text { Estádio de } \\
\text { repleção }\end{array}$ & $\begin{array}{c}\text { Índice } \\
\text { estádios } \\
\text { larvais } \\
\end{array}$ & $\begin{array}{l}\text { Estádio de } \\
\text { repleção }\end{array}$ \\
\hline 0 & I & & I & & I & & I & \\
\hline 2 & III & M & III & $\mathrm{M}$ & III & $\mathrm{V}$ & III & $\mathrm{V}$ \\
\hline 4 & III & M & IV & M & IV & M & III & M \\
\hline 6 & III & $\mathrm{V}$ & $\mathrm{V}$ & $\mathrm{C}$ & $\mathrm{V}$ & C & $\mathrm{V}$ & $\mathrm{C}$ \\
\hline 8 & $\mathrm{~V}$ & M & V & M & V & M & V & $\mathrm{M}$ \\
\hline 10 & IV & M & $\mathrm{V}$ & M & $\mathrm{V}$ & M & $\mathrm{V}$ & $\mathrm{M}$ \\
\hline 12 & V & M & VI & C & VI & $\mathrm{C}$ & V & M \\
\hline 14 & Morte & $\mathrm{V}$ & VI & M & VI & M & VI & M \\
\hline 16 & & & $\mathrm{VI}$ & M & $\mathrm{VI}$ & M & $\mathrm{VI}$ & M \\
\hline 19 & & & VII & $\mathrm{C}$ & VII & $\mathrm{C}$ & VII & M \\
\hline 21 & & & VII & M & VII & M & VII & M \\
\hline 23 & & & $\mathrm{X}$ & $\mathrm{C}$ & VIII & M & VIII & $\mathrm{C}$ \\
\hline 25 & & & $X$ & M & VIII & M & VIII & M \\
\hline 27 & & & $X$ & M & X & M & X & M \\
\hline 29 & & & $\mathrm{X}$ & $\mathrm{M}$ & $X$ & M & $X$ & $\mathrm{C}$ \\
\hline 31 & & & XI & $\mathrm{M}$ & $X$ & M & $X$ & $\mathrm{C}$ \\
\hline 33 & & & XI & $\mathrm{M}$ & $\mathrm{X}$ & $\mathrm{C}$ & $\mathrm{X}$ & M \\
\hline 35 & & & PL & $\mathrm{C}$ & PL & $\mathrm{C}$ & PL & $\mathrm{C}$ \\
\hline
\end{tabular}

*Estádio de repleção(repletion stadium): M=parcialmente vazio (partially empty), V=vazio (empty), C=cheio (full).

R. Bras. Zootec., v.33, n.6, p.1934-1941, 2004 (Supl. 2) 
Alguns autores sugerem que, a partir do VII estádio larval, as larvas capturam com maior facilidade o alimento, graças ao desenvolvimento do aparelho bucal dos apêndices de locomoção e por se tornarem omnívoras em maior grau, ou seja, menos seletivas em relação à ingestão e digestão dos alimentos (Lavens et al., 2000; Barros \& Valenti, 2003).

Neste contexto, realizando-se as análises estatísticas em relação à percentagem do índice do estádio larval e do estádio de repleção larval nos tratamentos
T1, T2, T3 e T4, em cada observação do $2^{\circ}$ ao $12^{\circ}$ dia, verificou-se que houve diferença significativa $(\mathrm{P}<0,05)$ no tratamento $\mathrm{T} 1$, em relação aos outros tratamentos em todas as observações (Tabelas 2 e 3 ).

O resultados obtidos (Tabelas 2 e 3 ) confirmam que o T1 apresentou o pior desempenho no desenvolvimento larval em relação aos demais, indicando que a larvicultura de $M$. rosenbergii não deve ser realizada com $100 \%$ de rotíferos nos 12 primeiros dias. Estes resultados corroboram com os relatos de Cor-

Tabela 2 - Porcentagem (\%) do índice do estágio larval (LSI) de M. rosenbergii, submetidos aos regimes

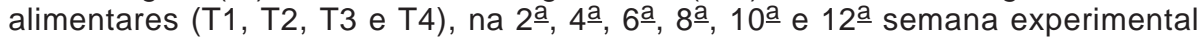

Table 2 - Percentage (\%) of the larval stage index (LSI) of M. rosenbergii, submitted to the feeding systems (T1, T2, T3 and T4), at the $2^{\text {nd }}, 4^{\text {th }}, 6^{\text {th }}, 8^{\text {th }}, 10^{\text {th }}$ and $12^{\text {th }}$ experimental observation

\begin{tabular}{|c|c|c|c|c|c|c|c|c|}
\hline \multicolumn{9}{|c|}{$\begin{array}{c}\text { Tratamentos } \\
\text { Treatments } \\
\end{array}$} \\
\hline & \multicolumn{2}{|c|}{$\begin{array}{c}\mathrm{T} 1 \\
(100 \% \text { Brachionus } \\
\text { plicatilis })\end{array}$} & \multicolumn{2}{|c|}{$\begin{array}{c}\mathrm{T} 2 \\
(100 \% \text { Artemia } s p)\end{array}$} & \multicolumn{2}{|c|}{$\begin{array}{c}\text { T3 } \\
(60 \% \text { Artemia sp. }+40 \% \\
\text { Brachionus plicatilis })\end{array}$} & \multicolumn{2}{|c|}{$\begin{array}{c}\mathrm{T} 4 \\
(40 \% \text { Artemia sp. }+60 \% \\
\text { Brachionus plicatilis })\end{array}$} \\
\hline Observação & Estádio & Animais (\%) & Estádio & Animais (\%) & Estádio & Animais (\%) & Estádio & Animais (\%) \\
\hline Observation & Stage & Animals & Stage & Animals & Stage & Animals & Stage & Animals \\
\hline 2 & III & $73^{\mathrm{b}}$ & III & $90^{\mathrm{a}}$ & III & $98^{\mathrm{a}}$ & III & $100^{\mathrm{a}}$ \\
\hline 4 & III & $70^{c}$ & IV & $68^{\mathrm{a}}$ & IV & $50^{\mathrm{a}}$ & III & $60^{\mathrm{bc}}$ \\
\hline 6 & III & $53^{\mathrm{b}}$ & $\mathrm{V}$ & $82^{\mathrm{a}}$ & $\mathrm{V}$ & $83^{a}$ & $\mathrm{~V}$ & $73^{a}$ \\
\hline 8 & $\mathrm{~V}$ & $47^{\mathrm{b}}$ & V & $50^{\mathrm{ab}}$ & $\mathrm{V}$ & $51^{\mathrm{ab}}$ & $\mathrm{V}$ & $52^{\mathrm{a}}$ \\
\hline 10 & IV & $45^{\mathrm{b}}$ & $\mathrm{V}$ & $52^{\mathrm{a}}$ & $\mathrm{V}$ & $51^{\mathrm{a}}$ & $\mathrm{V}$ & $50^{\mathrm{a}}$ \\
\hline 12 & $\mathrm{~V}$ & $53^{c}$ & VI & $58^{a}$ & VI & $56^{\mathrm{a}}$ & $\mathrm{V}$ & $54^{\mathrm{bc}}$ \\
\hline
\end{tabular}

${ }_{1}$ Médias seguidas de letras iguais na linha não diferem significativamente pelo teste de qui-quadrado a $5 \%$ de probabilidade. ${ }^{1}$ Mean followedg by the same letters in the rows do not differ significantly by chi-square test at $5 \%$ level probability.

Tabela 3 - Porcentagem (\%) do estádio de repleção larval de M. rosenbergii, submetidos aos regimes alimentares (T1,

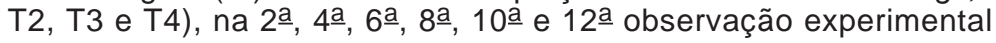

Table 3 - Percentage (\%) of the larval repletion stadium of M. rosenbergii, submitted to the feeding diets (T1, T2, T3 and T4), at the $2^{\text {nd }}, 4^{\text {th }}, 6^{\text {th }}, 8^{\text {th }}, 10^{\text {th }}$ and $12^{\text {th }}$ experimental observation

\begin{tabular}{|c|c|c|c|c|c|c|c|c|}
\hline \multirow[b]{3}{*}{ Observação } & \multicolumn{8}{|c|}{$\begin{array}{l}\text { Tratamentos } \\
\text { Treatments }\end{array}$} \\
\hline & \multicolumn{2}{|c|}{$\begin{array}{c}\mathrm{T} 1 \\
\text { (100\% Brachionus } \\
\text { plicatilis) }\end{array}$} & \multicolumn{2}{|c|}{$\begin{array}{c}\mathrm{T} 2 \\
(100 \% \text { Artemia } s p)\end{array}$} & \multicolumn{2}{|c|}{$\begin{array}{c}\text { T3 } \\
(60 \% \text { Artemia sp. }+40 \% \\
\text { Brachionus plicatilis) }\end{array}$} & \multicolumn{2}{|c|}{$\begin{array}{c}\text { T4 } \\
(40 \% \text { Artemia sp. }+60 \% \\
\text { Brachionus plicatilis })\end{array}$} \\
\hline & Repleção & Animais (\%) & Repleção & Animais (\%) & Repleção & Animais (\%) & Repleção & Animais (\%) \\
\hline Observation & Repletion & Animals & Repletion & Animals & Repletion & Animals & Repletion & Animals \\
\hline 2 & M & $73^{\mathrm{b}}$ & M & $90^{\mathrm{a}}$ & $\mathrm{V}$ & $98^{\mathrm{a}}$ & $\mathrm{V}$ & $100^{\mathrm{a}}$ \\
\hline 4 & M & $70^{c}$ & M & $68^{\mathrm{a}}$ & M & $50^{\mathrm{a}}$ & M & $60^{\mathrm{bc}}$ \\
\hline 6 & $\mathrm{~V}$ & $53^{\mathrm{b}}$ & $\mathrm{C}$ & $82^{a}$ & $\mathrm{C}$ & $83^{a}$ & $\mathrm{C}$ & $73^{a}$ \\
\hline 8 & M & $47^{\mathrm{b}}$ & M & $50^{\mathrm{ab}}$ & M & $51^{\mathrm{ab}}$ & M & $52^{\mathrm{a}}$ \\
\hline 10 & M & $45^{\mathrm{b}}$ & M & $52^{\mathrm{a}}$ & M & $51^{\mathrm{a}}$ & M & $50^{\mathrm{a}}$ \\
\hline 12 & $\mathrm{~V}$ & $53^{c}$ & M & $58^{\mathrm{a}}$ & $\mathrm{M}$ & $56^{\mathrm{a}}$ & M & $54^{\mathrm{bc}}$ \\
\hline
\end{tabular}

${ }^{1}$ Médias seguidas de letras iguais na linha não diferem significativamente pelo teste de qui-quadrado a $5 \%$ de probabilidade.

${ }^{1}$ Mean followedg by the same letters in the rows do not differ significantly by chi-square test at $5 \%$ level probability.

Repleção(repletion): M=parcialmente vazio (partially emptiness), V=vazio (empty), C=cheio (full).

\section{R. Bras. Zootec., v.33, n.6, p.1934-1941, 2004 (Supl. 2)}


reia et al. (2000) e New (2000), que afirmam que camarão-d'água-doce não absorvem eficazmente, durante os primeiros estádios larvais, dietas artificiais e outras fontes alternativas de alimentos vivos.

Estes resultados discordam daqueles descritos por Seixas Filho et al. (2000), que realizaram a substituição parcial dos náuplios de Artemia sp., pelo rotífero $B$. plicatilis na densidade de 30 por $\mathrm{mL}$, enriquecido com levedura e emulsão comercial de óleo de fígado de bacalhau por 12 horas e congelado. Estes autores verificaram que o enriquecimento de rotíferos com ácidos graxos poliinsaturados e seu fornecimento até o sexto estádio larval de $M$. rosenbergii (10 dias) proporcionaram desempenho satisfatório quanto à sobrevivência entre as larviculturas com substituição parcial de Artemia sp. e tratamento-controle.

Trabalhos têm demonstrado as distintas propriedades no desenvolvimento larval inicial e as limitações quanto à captura, ingestão e digestão dos alimentos até o estádio larval VI (Barros \& Valenti, 1997; 2003). As larvas são dependentes de alimentos vivos, podendo ingerir rapidamente os náuplios recém-eclodidos de Artemia sp., que podem prover enzimas exógenas que conduzem a satisfação de seus requerimentos nutricionais (Lavens et al., 2000).

A substituição parcial (T3 e T4) da alimentação tradicional (T2) ao final do período experimental não apresentou diferença significativa $(\mathrm{P}>0,05)$ entre os tratamentos (T2, T3 e T4) analisados pelo teste do qui-quadrado (Tabela 4). Portanto, o rotífero enriquecido (B. plicatilis) pode substituir parcialmente a alimentação tradicional (Artemia sp.), podendo ser viável se considerado o custo destes alimentos vivos.

Segundo Galvão et al. (1998.) a utilização eficiente de $B$. plicatilis depende do sucesso da técnica de enriquecimento, capaz de afetar a composição bioquímica dos rotíferos (B. plicatilis). Este experimento sugere que o enriquecimento de rotíferos, utilizando solução de fermento biológico (Saccharomyces cerevisae) e a microalga (Nanocloropsi oculata) + emulsão de óleo de fígado de bacalhau (preparo comercial) por 12 horas sobre o meio de cultivo, pode ter aumentado a porcentagem de lipídios totais dos rotíferos, atingindo uma média superior aos da Artemia sp., especialmente os derivados de W3, tornando-os transportadores de vários produtos essenciais na alimentação de larvas de crustáceos (Barreto \& Cavalcanti, 1997).

No entanto, estudos futuros são necessários para aprimoramento das técnicas de avaliação do desempenho das larvas de camarão-d'água-doce para redução de custos operacionais na criação e, conseqüentemente, maior autonomia na produção larval.

Tabela 4 - Porcentagem (\%) do índice do estádio larval (LSI) e do estádio de repleção de $M$. rosenbergii, submetidos aos regimes alimentares (T2, T3 e T4), ao final do período experimental

Table 4 - Percentage (\%) of the larval stage index (LSI) and repletion stadium of M. rosenbergii, submitted to the feeding systems (T2, T3 and T4), at the end of the experimental period

\begin{tabular}{lccc}
\hline $\begin{array}{l}\text { Tratamento } \\
\text { Treatments }\end{array}$ & $\begin{array}{c}\text { Estádio } \\
\text { Stage }\end{array}$ & $\begin{array}{c}\text { Repleção } \\
\text { Repletion }\end{array}$ & LSI \\
\hline T2 (100\% Artemia) & PL & $C^{\text {ns }}$ & $91^{\text {ns }}$ \\
T3(60\% Artemia + 40\% Rotífero) & PL & $C^{\text {ns }}$ & $90^{\text {ns }}$ \\
T4(40\% Artemia + 60\% Rotífero) & PL & $C^{\text {ns }}$ & $90^{\text {ns }}$ \\
\hline
\end{tabular}

ns = não-significativo pelo teste qui-quadrado a $5 \%$ de probabilidade.

$n s=$ not significant by chi-square test at $5 \%$ level probability.

LSI = Índice de estádio larval.

$L S I=$ Larval stage index .

$\mathrm{PL}=$ Pós-larva .

$P L=$ Post-larvae.

$\mathrm{C}=$ cheio.

R. Bras. Zootec., v.33, n.6, p.1934-1941, 2004 (Supl. 2) 


\section{Conclusões}

A técnica de avaliação do desempenho das larvas de $M$. rosenbergii utilizando a observação diária dos subestádios larvais do índice dos estádios larvais e do índice do estádio de repleção foi satisfatória.

O rotífero B. plicatilis enriquecido e congelado não pode substituir totalmente os náuplios de Artemia sp., na larvicultura de $M$. rosenbergii.

A utilização de B. plicatilis enriquecido e congelado apresentou resultado satisfatório no desempenho das larvas, podendo substituir parcialmente os náuplios de Artemia sp., na proporção de 40 ou $60 \%$, no regime alimentar das larvas de $M$. rosenbergii.

\section{Literatura Citada}

ALAM, M.J.; CHEAH, S.H.; ANG, K.J. Possible use of Moina spp. as a live feed substitute in larval rearing of the freshwater prawn, Macrobrachium rosenbergii (De Man). Aquaculture Fishery Management, v.22, n.4, p.531-535, 1991.

ALAM, M.J; ANG, K.J.; CHEAH, S.H. Use of Moina micrura (Kurz) as an Artemia substitute in the production of. Macrobrachium rosenbergii (De Man) post-larvae. Aquaculture, v.109, p.337-349, 1993a.

ALAM, M.J.; ANG, K.J.; CHEAH, S.H. Weaning of Macrobrachium rosenbergii (De Man) larvae from Artemia to Moina micrura (Kurz). Aquaculture, v.12, p.187-194, 1993b.

BARRETO, O.J.S.; CAVALCANTI, D.G. Enriquecimento de alimentos vivos para alimentação de larvas de organismos marinhos: uma breve revisão. Boletim do Instituto de Pesca, v.24, p.139-159, 1997.

BARROS, H.P.; VALENTI, W.C. Comportamento alimentar do camarão de água doce, Macrobrachium rosenbergii (De Man, 1879) (CRUSTACEA, PALAEMONIDAE) durante a fase larval: análise quantitativa. Revista Brasileira de Zoologia, v.14, n.4, p.785-793, 1997.

BARROS, H.P.; VALENTI, W.C. Ingestion rates of Artemia nauolii for different larval stages of Macrobrachium rosenbergii. Aquaculture, v.217, n.(1-4), p.223-233, 2003.

CORREIA, E.S.; SUWANNATOUS, S.; NEW, M. Flowthrough hatchery systems and management In: NEW, M.B.; VALENTI, W.C. (Eds.) Freshwater prawn culture: the farming of Macrobrachium rosenbergii. Oxford: Blackwell, 2000. p.52-68.

DANIELS, W.H.; D’ ABRAMO, L.R.; DE PARSEVAL, L. Design and management of closed, recirculating clearwater hatchery system for freshwater prawns Macrobrachium rosenbergii. Journal of Shellfish Research, v.11, p.65-73, 1992.

FOOD AND AGRICULTURE ORGANIZATION OF THE UNITED NATIONS - FAO. 2002. Yearbook of fishery statistics: summary tables. FAO, Roma (obtido via internet, http://www.fao.org).
GALVÃO, M.S.N.; BARRETO, O.J.S.; YAMANAKA, N. Cultivo intensivo dos rotíferos Brachionus plicatilis $\mathbf{O}$. F. Muller, 1786 e Brachionus rotundiformis Tschugunoff, 1921 (Rotifera, Brachionidae). São Paulo: 1988. v.24, 15p.

LAI, L. L. Production methods and role of the brine shrimp Artemia. Belgium: Academic Press, 1985. 267p.

LAVENS, P.; THONGROD, S.; SORGELOOS. P. Larval prawn feeds and the dietary importance of Artemia In: NEW, M.B.; VALENTI, W.C. (Eds.) Freshwater prawn culture: the farming of Macrobrachium rosenbergii. Blackwell: Oxford, 2000. p.91-111.

LOVETT, D.L.; FELDER, D.L. Evaluation of. the rotifer Brachionus plicatilis as a substitute for Artemia sp. in feeding larvas of Macrobrachium rosenbergii. Aquaculture, v.71, p.331-338, 1988.

NEW, M.B. Freshwater praw culture: a review. Aquaculture, v.88, p.99-143, 1990.

NEW, M.B. Status of freshwater prawn farming: a review. Aquaculture Research, v.26, n.1, p.1-54, 1995.

NEW, M.B. History and global status of freshwater prawn farming. In: NEW, M.B.; VALENTI, W.C. (Eds.) Freshwater prawn culture: the farming of Macrobrachium rosenbergii. Oxford: Blackwell, 2000. p.01-11.

RODRIGUES, C.C.; RODRIGUES, J.B.R.; MOSCHEN, S. Efeito da luz solar e cor dos tanques no desenvolvimento larval e produção de pós-larvas do camarão Macrobrachium rosenbergii (De Man, 1879). In: SIMPÓSIO BRASILEIRO DE AQÜICUltura, 10., 1998, Recife. Anais... Pernambuco: ABRAq, 1998. p.423-435.

SEIXAS FILHO, J.T.; SIMÃO, O.M.; TRIANI, L. et al. Rotífero: uma alternativa no arraçoamento larval de Macrobrachium rosenbergii. [C.T.]: Pesagro, 1984. p.1-3.

SEIXAS FILHO, J.T.; TRIANI, L.; THOMAZ, L.A. et al. Utilização da morfometria na avaliação de larvas do camarão de água doce Macrobrachium rosenbergii (De Man, 1879) submetidas a diferentes regimes alimentares. In: SIMPÓSIO BRASILEIRO DE AQÜICULTURASimbraq, 11., 2000, Florianópolis. Anais... Santa Catarina: SIMBRAq, 2000. p.2-17.

SILVA, F.M.; RODRIGUES, J.B.R. Efeito da substituição de Artemia sp. pelo Nematóide Panagrellus redivivus sobre o crescimento e sobrevivência larval do camarão de água doce. Boletim do Instituto de Pesca, v.24 (especial), p.35-48, 1997.

SORGELOOS, P.; BOSSUYT, E.; LAVENS, P. et al. The use of brine shrimp Artemia in crustacean hatcheries and nurseries. In: Mc VEY, J.P. (Ed.) Handbook of. Maricult. I. vol. Crustacean Aquaculture: Florida, 1983. p. 71-96.

SORGELOOS, P.; LÉGER, P. Improved larviculture outputs of marine fish, shrimp and prawn. Journal World Aquaculture Society, v.23, n.4, p.251-264, 1992.

STOTTRUP, J.G.; ATTRAMADAL, Y. The influence of different rotifer and Artemia enrichment diets on growth, survival and pigmentation in turbot (Scophthlmus maximus L.) larvae. Journal World Aquaculture, v.3, n.4, p.307315, 1992.

UNO, Y.; SOO, K. C. Larval development of. Macrobrachium rosenbergii reared in the laboratory. Journal Tokyo Fish, v.55, p.179-190, 1969. 
VALENTI. W. C. Criação de camarão da Malásia. Jaboticabal: FUNEP, 1991. 53p.

VALENTI, W.C. Carcinicultura de água doce. Jaboticabal: FUNEP, 1998. 383p.

VALENTI, W.C. A modernização da carcinicultura de água doce. Revista da Associação Brasileira dos Criadores de Camarões, ano III, $\mathrm{n}^{\circ}$ 1, abril de 2001. p.56-58.

WATANABE, T.; IZQUIERDO, M.S.; TAKEUCHI, T. et al. Comparison between eicosaopentaenoic and docosahexaenoic acids in terms of essential fatty acid efficacy in larval red sea bream. Nippon Suisan Gakkaishi, v.55, n.9, p.1635-1640, 1989.
YUFERA, M.; RODRIGUEZ, A.; LUBIAN, L.M. Zooplankton ingestion and feeding behavior of Penaeus kerathurus larvae reared in the laboratory. Aquaculture, v.42, p.217-224, 1985.

Recebido em: 07/11/02

Aceito em: 12/11/03 